Article

\title{
Monitoring the Interfacial Polymerization of Piperazine and Trimesoyl Chloride with Hydrophilic Interlayer or Macromolecular Additive by In Situ FT-IR Spectroscopy
}

\author{
Xi Yang \\ Department of Polymer Science \& Engineering, Zhejiang University, Hangzhou 310027, China; \\ 11529019@zju.edu.cn; Tel.: +86-187-5810-1644
}

Received: 31 October 2019; Accepted: 6 January 2020; Published: 7 January 2020

\begin{abstract}
The interfacial polymerization (IP) of piperazine (PIP) and trimesoyl chloride (TMC) has been extensively utilized to synthesize nanofiltration (NF) membranes. However, it is still a huge challenge to monitor the IP reaction, because of the fast reaction rate and the formed ultra-thin film. Herein, two effective strategies were applied to reduce the IP reaction rate: (1) the introduction of hydrophilic interlayers between the porous substrate and the formed polyamide layer, and (2) the addition of macromolecular additives in the aqueous solution of PIP. As a result, in situ Fourier transform infrared (FT-IR) spectroscopy was firstly used to monitor the IP reaction of PIP/TMC with hydrophilic interlayers or macromolecular additives in the aqueous solution of PIP. Moreover, the formed polyamide layer growth on the substrate was studied in a real-time manner. The in situ FT-IR experimental results confirmed that the IP reaction rates were effectively suppressed and that the formed polyamide thickness was reduced from $138 \pm 24 \mathrm{~nm}$ to $46 \pm 2 \mathrm{~nm}$ according to TEM observation. Furthermore, an optimized NF membrane with excellent performance was consequently obtained, which included boosted water permeation of about 141-238 (L/m².h.MPa) and superior salt rejection of $\mathrm{Na}_{2} \mathrm{SO}_{4}>98.4 \%$.
\end{abstract}

Keywords: interfacial polymerization; in situ FT-IR spectroscopy; thin-film composite membrane; nanofiltration membrane

\section{Introduction}

The interfacial polymerization (IP) method has been widely employed to fabricate polyamide-based membranes with a thin-film composite (TFC) structure, which include top polyamide layer, middle ultrafiltration support, and bottom non-woven fabric. TFC membranes are extensively used in the waste-water treatment field of reverse osmosis (RO) [1,2], nanofiltration (NF) [3], forward osmosis (FO) $[4,5]$, and gas separation (GS). As it is reported in the literature, the IP process usually takes place at the interface of two immiscible solvents, such as the water/hexane interface. Typical reactive monomers of diamine and acid chloride monomers are separately dissolved in the solvents [6]. The diamine monomer is able to diffuse into the organic phase and subsequently reacts inside the organic phase near the interface, and then immediately forms the polyamide thin film in situ at the interface within several seconds [7]. As a consequence, the IP reaction has been successfully utilized to synthesize an ultra-thin and dense polyamide layer on the top surface on a porous substrate, which serves both as the storage location of the diamine monomer, and as the support of the formed ultra-thin polyamide film $[8,9]$.

The IP reaction usually has a rapid reaction rate of about $10^{4} \mathrm{~mol} /(\mathrm{L} \cdot \mathrm{s})$, and the thickness of the formed ultra-thin film is normally less than $100 \mathrm{~nm}$ [10]. Therefore, it is a huge challenge to monitor the IP reaction in a real-time manner, as well as investigating the IP reaction kinetics directly on the 
porous substrates [10]. To the best of our knowledge, few methods have been developed to study the IP reaction, where the polyamide film is formed at the free water/oil interface. It is possible for one to terminate the IP reaction at different times, including determining the film mass [11], measuring the film thickness [12], and/or analyzing the reactant concentration [13-15]. Furthermore, the polyamide film formation at the free water/oil interface has also been observed by measuring the suspended drop interfacial tension [16], light reflection [16], and diffusion reflectance spectra [17]. Matthews et al. used Rutherford backscattering spectrometry (RBS) to study the polyamide growth dynamics, and established a relationship between the diffusion reflectance and the polyamide layer thickness [17]. Recently, Nowbahar et al. applied the microfluidic interferometry to measure the IP reaction kinetics of the m-phenylenediamine (MPD)/trimesoyl chloride (TMC) reaction system [10]. Although these techniques can be used to roughly estimate the IP reaction, they are still limited to detecting the IP reaction at the free water/oil interface, instead of directly on the porous substrate. Therefore, it is of great significance to develop effective strategies for monitoring the IP reaction, which takes place on the substrate in a real-time manner.

Herein, we report in situ Fourier transform infrared (FT-IR) spectroscopy as an effective instrument to monitor the IP reaction and the polyamide film growth on the porous substrate in real-time. In situ FT-IR spectroscopy is a powerful and well-established tool to investigate the reaction taking place at the solid/liquid interface $[18,19]$, and to provide the real-time information about the IP reaction, such as mechanism and kinetics [20,21]. For example, Han et al. used a self-made FT-IR sample cell to monitor the photo-polymerization of alicyclic methacrylate hydrogel for controlled drug release [22]. Zimudzi et al. analyzed the Nafion film thickness [23], and quantified the carboxylic acid concentration of the polyamide-based membrane [24]. Moreover, it is reasonable to associate the absorbance intensity of characteristic band in the FT-IR spectra with the polyamide film thickness by mathematical equations [25-28]. Additionally, Ren et al. used FT-IR microscopy for IP reaction of microporous polymer film formed in the aqueous/organic interface for polyesterification by choosing the adequate phenol monomer [29]. Yang et al. reduced the IP reaction rate of piperazine (PIP) and trimesoyl chloride (TMC) reaction system to a certain extent by introducing interlayers between the porous substrate and the polyamide layer [30-32]. Tan et al. added the macromolecular additive of polyvinyl alcohol (PVA) in the aqueous solution of PIP and obtained the Turing structure [33]. Furthermore, in situ FT-IR spectroscopy is used to measure the IP reaction in real-time. The relationship between the IP reaction rate and the polyamide film thickness was successfully established for laying a foundation to optimize the NF membrane performance. The fabricated NF membranes exhibited boosted water permeation of about $141-238\left(\mathrm{~L} / \mathrm{m}^{2} \cdot \mathrm{h} \cdot \mathrm{MPa}\right)$ and superior salt rejection of $\mathrm{Na}_{2} \mathrm{SO}_{4}>98.4 \%$. These improved NF membrane performances were attributed to the reduced polyamide layer thickness and the remaining high cross-linking degree of the internal structure of the polyamide layer.

\section{Materials and Methods}

\subsection{Materials}

Polysulfone (PSf) ultrafiltration membranes with a molecular weight cut-off (MWCO) of $50 \mathrm{kDa}$ were used as the porous substrates, and were purchased from Ande Membrane Separation Technology and Engineering Co., Ltd. (Beijing, China). Dopamine hydrochloride (DA), tris-(hydroxymethyl) aminomethane (Tris), and N,N-bis(2-hydroxyethyl)glycine (Bicine) were obtained from Sigma-Aldrich (Shanghai, China). Polyethyleneimine (PEI, $\mathrm{M}_{\mathrm{W}} \approx 600 \mathrm{Da}$ ), tannic acid (TA), polyethyleneglycol (PEG, $\mathrm{M}_{\mathrm{W}}=20,000$ ), polyvinylpyrrolidone (PVP, K23-27, $\mathrm{M}_{\mathrm{w}} \approx 24,000$ ), polyvinyl alcohol (PVA 1788, $\mathrm{M}_{\mathrm{w}}$ 75,000-80,000, 87\%-89\% hydrolyzed), and piperazine (PIP) were purchased from Aladdin Chemical Co., Ltd. (Shanghai, China). Trimesoyl chloride (TMC) was procured from Qingdao Benzo Chemistry Co., Ltd. (Qingdao, China). 2-Methylimidazole (Hmim) was purchased from Tokyo Chemical Industry Co., Ltd. (Tokyo, Japan). Other chemicals, including $\mathrm{Zn}\left(\mathrm{NO}_{3}\right)_{2} \cdot 6 \mathrm{H}_{2} \mathrm{O}, n$-hexane, ethanol, and inorganic salts $\left(\mathrm{Na}_{2} \mathrm{SO}_{4}, \mathrm{MgSO}_{4}, \mathrm{MgCl}_{2}, \mathrm{CaCl}_{2}\right.$, and $\left.\mathrm{NaCl}\right)$ were bought from Sinopharm Chemical Reagent $\mathrm{Co}$., 
Ltd. (Shanghai, China). Ultra-pure water consumed in the experiments was directly generated from a lab water purification system (HYP-QX, Hangzhou, China).

\subsection{Fabrication of Interlayers on the Porous Substrate}

The porous substrates were cut into circular shape with a diameter of $47 \mathrm{~mm}$ and rinsed in deionized (DI) water and ethanol overnight. Various interlayers were fabricated on the porous substrate, including polydopamine (PDA)/polyethyleneimine (PEI), tannic acid (TA)/polyethyleneimine (PEI), and zeolitic imidazolate framework-8 (ZIF-8)/polyethyleneimine (PEI).

The PDA/PEI interlayer was constructed on the porous substrate, according to the reported literature. DA and PEI were dissolved in Tris-buffer $(50 \mathrm{mM}, \mathrm{pH}=8.5)$ at a mass ratio of $1: 1$, with a concentration of $2.0 \mathrm{mg} / \mathrm{mL}$ [30]. The porous substrate was immersed in the freshly prepared PDA/PEI solution for $1 \mathrm{~h}$. After that, the PDA/PEI modified substrate was washed thoroughly by DI water and dried at room temperature for $1 \mathrm{~h}$.

The TA/PEI interlayer was fabricated on the porous substrate via Michael addition or Schiff base reaction between the amino groups and quinone groups [31]. TA $(2.0 \mathrm{mg} / \mathrm{mL})$ was dissolved in a Bicine buffer solution ( $\mathrm{pH}=7.8)$, then PEI $(2.0 \mathrm{mg} / \mathrm{mL})$ was added in the solution. The substrate was immersed in the freshly prepared TA/PEI solution for $1 \mathrm{~h}$, followed by washing procedure with DI water and drying at room temperature for $1 \mathrm{~h}$ of the TA/PEI modified substrate.

The ZIF-8/PEI interlayer was constructed on the porous substrate with the following steps [34,35]. The porous substrate was immersed in an equal volume of $0.1 \mathrm{~mol} / \mathrm{L} \mathrm{Zn}\left(\mathrm{NO}_{3}\right)_{2}$ aqueous solution and 0.4 wt \% PEI aqueous solution for $0.5 \mathrm{~h}$ at $25 \pm 2{ }^{\circ} \mathrm{C}$. Next, the substrate was rinsed with DI water to remove the excess residual PEI molecules. Then, the porous substrate was immersed in $0.2 \mathrm{~mol} / \mathrm{L}$ Hmim in $n$-hexane solution, which reacted with $\mathrm{Zn}^{2+}$ for $0.5 \mathrm{~h}$. The as-prepared ZIF-8/PEI modified substrate was rinsed with DI water and dried at room temperature for further usage.

\subsection{IP on the Substrates with Modified Interlayers}

The porous substrates modified with as-formed interlayers were upper-side immersed in $10 \mathrm{~mL}$ aqueous solution of $2.0 \mathrm{~g} / \mathrm{L}$ PIP for $10 \mathrm{~min}$, ensuring the complete adsorption and infiltration of diamine solution on the interlayer surfaces and the substrate internal pores. A rubber roller was gently used to thoroughly dry the excess PIP solution on the top surface. Then, $10 \mathrm{~mL}$ of TMC solution with a concentration of $2.0 \mathrm{~g} / \mathrm{L}$ in $n$-hexane was carefully poured on the substrates. Therefore, IP reaction was then consequently carried out for about $100 \mathrm{~s}$ of the optimum IP reaction time, to form the ultra-thin polyamide layers on top surfaces of the porous substrates. The resulted membranes were post-treated in an oven for $30 \mathrm{~min}$ at $60^{\circ} \mathrm{C}$, in order to further solidify and stabilize the formed polyamide layer.

\subsection{IP on the Substrates with Macromolecular Additives}

Macromolecular additives of PEG, PVP, and PVA were added into the aqueous solution of PIP. The aqueous solutions were prepared by mixing $1.5 \mathrm{~g} / \mathrm{L}$ of macromolecular additive evenly with $2 \mathrm{~g} / \mathrm{L}$ of PIP. The aqueous solutions were stirred at $25 \pm 2{ }^{\circ} \mathrm{C}$ for $1 \mathrm{~h}$ and rested for $30 \mathrm{~min}$ to be uniformly mixed and to eliminate air bubbles. Then the IP reaction was then conducted on the porous substrates as per the method above. The resulting membranes were post-treated in an oven for $30 \mathrm{~min}$ at $60{ }^{\circ} \mathrm{C}$, in order to further solidify and stabilize the formed polyamide layer.

\subsection{Measuring IP Reaction by In Situ FT-IR Spectroscopy}

Polyamide formation on the porous substrates was measured by in situ FT-IR spectroscopy (React 15, Mettler Toledo, Switzerland), with the scan range between $4000-650 \mathrm{~cm}^{-1}$, the maximum resolution of $4 \mathrm{~cm}^{-1}$, and the minimum detection time interval of $15 \mathrm{~s}$. During the detection process, one sample of the porous substrate was fixed in a home-made reactor to ensure the upper-side made contact with the monomer solutions of PIP and TMC. Firstly, the FT-IR baseline was set up in the air environment, and then $10 \mathrm{~mL}$ of PIP solution $(2.0 \mathrm{~g} / \mathrm{L})$ was poured onto the substrate surface for $10 \mathrm{~min}$. The PIP 
solution was then poured off thoroughly and wiped by filter paper to remove the excess PIP solution on the surface. An optic probe was tightly contacted with the middle area of the substrate surface. Then, $10 \mathrm{~mL}$ of TMC solution $(2.0 \mathrm{~g} / \mathrm{L})$ was poured on the substrate surface for conducting the IP reaction for a certain time. Data collection instantaneously began by the in situ FT-IR spectroscopy monitoring process. Subsequently, the FT-IR absorbance intensity was acquired and calculated from the polyamide film formation as a function of IP reaction time. In addition, measuring the polyamide layer thicknesses through FT-IR via reference [S3] in the Supplementary Information was only demonstrated for dense uniform films and, although this is not the case for IP-based membranes, it could still yield valid information on the IP process. In the calculation in the Supplementary Information, it is necessary to assume the polymer density to be 1.5, even though this is approximate, because the density is not constant throughout a PA film, nor throughout the interfacial polymerization reaction time.

\subsection{Other Characterizations}

Surface morphologies were observed by field emission scanning electron microscopy (FESEM, Hitachi, SU-8010, Japan) for the porous substrates and the formed polyamide layer. Topographies and surface roughness were measured by atomic force microscopy (AFM, MultiMode, Vecco, USA) in the tapping mode. Transmission electron microscopy (TEM, Hitachi 7650, Japan) was applied to observe the cross-sectional morphologies and to measure the thickness of polyamide layer by the microtomy sections. The samples were embedded in LR white resin (London Resin Company, Reading, UK), cut by ultramicrotome (Leica Microsystems, Wetzlar, Germany), and then mounted to copper grids. The crystalline structure of ZIF-8 was analyzed by an X-Ray diffractometer (XRD, 7000S/L, Shimadzu, Japan), using $\mathrm{Cu} \mathrm{K} \alpha$ radiation, in the interval of $5^{\circ} \leq 2 \theta \leq 50^{\circ}$, with simulated pattern was generated from CIF files of ZIF-8. Chemical structures were analyzed by attenuated total reflectance Fourier transform infrared spectrometry (ATR/FT-IR, Nicolet 6700, USA) and X-ray photoelectron spectrometry (XPS, Thermo Scientific, USA). Surface hydrophilicity was measured by a contact angle measurement system (Surface-Meter, OCA 200, China), and each sample was measured three times to get the average value and deviations. The surface zeta potential was evaluated by an electro kinetic analyzer (SurPASS Anton Paar, GmbH, Austria).

\subsection{NF Membrane Performance Evaluation}

NF membrane performance of the as-formed membranes were evaluated by a laboratory scale cross-flow flat module. The effective filtration area was $7.07 \mathrm{~cm}^{2}$ and the cross-flow rate was $30 \mathrm{~L} / \mathrm{h}$ under the operation pressure of $0.6 \mathrm{MPa}$ at room temperature for $1 \mathrm{~h}$ reaching the steady state. Various inorganic salts, including $\mathrm{Na}_{2} \mathrm{SO}_{4}, \mathrm{MgSO}_{4}, \mathrm{MgCl}_{2}, \mathrm{CaCl}_{2}$, and $\mathrm{NaCl}$, were dissolved in $\mathrm{DI}$ water at a concentration of $1000 \mathrm{mg} / \mathrm{L}$ to prepare the feed solutions. The water permeation $\left(J_{w}, \mathrm{~L} / \mathrm{m}^{2} \cdot \mathrm{h}\right)$ and the salt rejection $(R, \%)$ were calculated by the following equations:

$$
J_{w}=\frac{V}{A \cdot t}
$$

where $V(\mathrm{~L}), A\left(\mathrm{~m}^{2}\right)$, and $t(\mathrm{~h})$ represent the filtered water volume, the effective membrane area, and the permeation time, respectively.

$$
R=\left(1-\frac{C_{p}}{C_{f}}\right) \times 100 \%
$$

where $C_{p}$ and $C_{f}(\mathrm{mg} / \mathrm{L})$ are the feed and permeated solution concentrations, respectively, determined by an electrical conductivity meter (Mettler Toledo, FE30, Hangzhou, China) for average value as a result of three repeated measurements.

\section{Results and Discussion}

A typical IP reaction is usually finished in $0.5-2.0 \mathrm{~min}$, because of the fast and uncontrollable IP reaction. In this work, hydrophilic interlayers and macromolecular additives were used to reduce 
the IP reaction rate for in situ FT-IR spectroscopy analyzing (as schematically shown in Figure 1). In addition, ATR/FT-IR spectra validated the formation of interlayers including PDA/PEI, TA/PEI, and ZIF-8/PEI on the porous substrates (Figure S1 in the Supporting Information). As a result, the formed interlayers reasonably changed the surface morphology, surface hydrophilicity, and surface charge of the porous substrates (Figures S2-S6 in the Supporting Information).

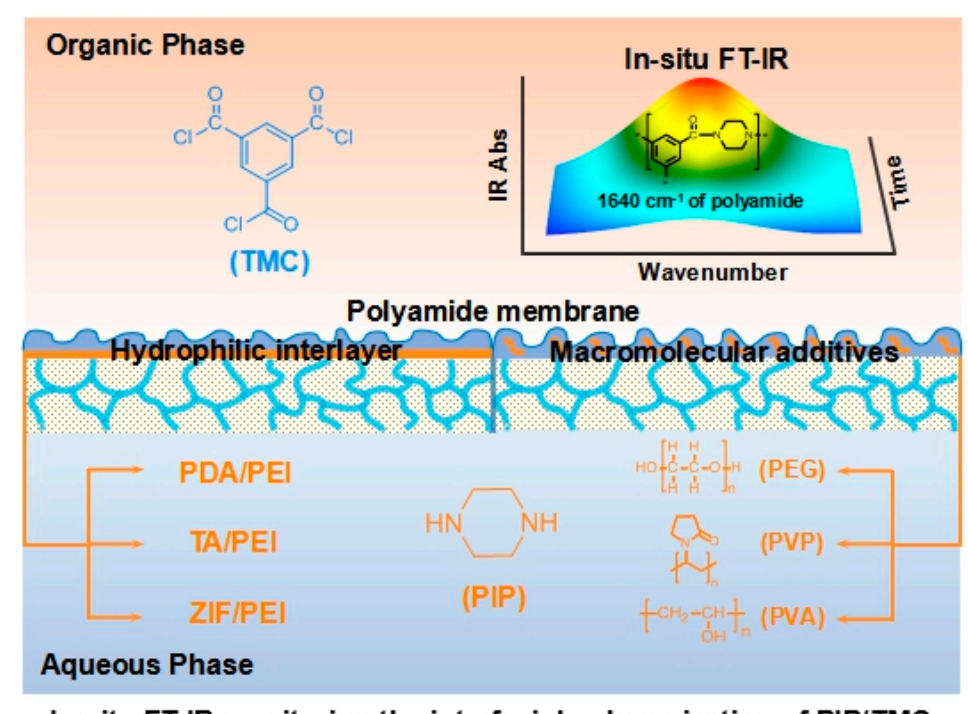

Figure 1. In-situ Fourier transform infrared (FT-IR) spectroscopy monitoring the interfacial polymerization (IP) reaction of piperazine (PIP)/trimesoyl chloride (TMC) by the introduction of hydrophilic interlayers on the pristine polysulfone (PSf) substrate or macromolecular additives in the aqueous solution of PIP. PDA: polydopamine (PDA), PEI: polyethyleneimine, TA: tannic acid, ZIF: zeolitic imidazolate framework, PEG: polyethyleneglycol, PVP: polyvinylpyrrolidone, PVA: polyvinyl alcohol.

Figures 2 and 3 show the in situ FT-IR spectra monitoring the IP reaction on the porous substrates of $\mathrm{C}=\mathrm{O}$ stretching vibration, specifically shown at $1640 \mathrm{~cm}^{-1}$. The absorbance intensities are also shown in Figure S7 in the Supporting Information, as an indicator of the polyamide formation process. Here, I took the carbonyl vibration at $1640 \mathrm{~cm}^{-1}$. However, this was the carbonyl vibration of the amide, and as such there will be an error depending on the variation in chemical structure which was based on the following XPS analysis, because the polyamide structure was heterogeneous in the polyamide layer depth [36,37]. Figure 2 shows that the in situ FT-IR spectra monitored the IP reaction on the pristine PSf porous substrate, which nicely complied with the traditional growth principle. Usually, in situ FT-IR can be used to monitor a reaction for several hours; in this case of a fast IP reaction, the monitoring time was fixed between 0-300 s, with the minimum interval of $15 \mathrm{~s}$. In other circumstances, the IP reaction rates are obviously repressed, in the condition of the introduction with hydrophilic interlayers and/or macromolecular additives. The reason for the repression phenomena could be possibly attributed to the slowed-down PIP diffusion rates, which were studied and acquired from the ultraviolet spectroscopy (UV-VIS) analyses for the 10 min diffusion time of PIP from water to hexane, as shown in Figure S8 in the Supporting Information. As a result, the measured PIP diffusion rates are commendably in accordance with the decreased diffusion co-efficient reported in the literature [33], which reduced from about $10^{-5}$ to $10^{-6} \mathrm{~cm}^{2} / \mathrm{s}$, as shown in Table S1 in the Supporting Information.

Furthermore, the reduced IP reaction rates also arose from the combination results of enhanced storage ability of PIP in the interlayers on the modified PSf substrate, and the enhanced interactions between the PIP and interlayers (Table S2 and Figure S9 in the Supporting Information). In detailed analysis, the adhesive PDA/PEI and TA/PEI interlayers impeded the IP reaction rate and maintained a similar growth tendency compared with the IP reaction on the pristine PSf substrate. Additionally, polyamide growth on the ZIF-8/PEI interlayer obeyed the approximately painful and linear propensity, 
as the growth rate was nearly maintained in an unchanged manner during the IP reaction. The reason behind this was that ZIF-8/PEI is a high-porosity and inorganic interlayer, which restores and reduces PIP diffusion greatly. As for the doped polyamide layer with the macromolecular additives, polyamide growth was considerably repressed because of the low flow viscosity of macromolecular additives and their strong hydrogen-bonding interactions with the PIP monomer (as shown in Figures S10 and S11 in the Supporting Information).

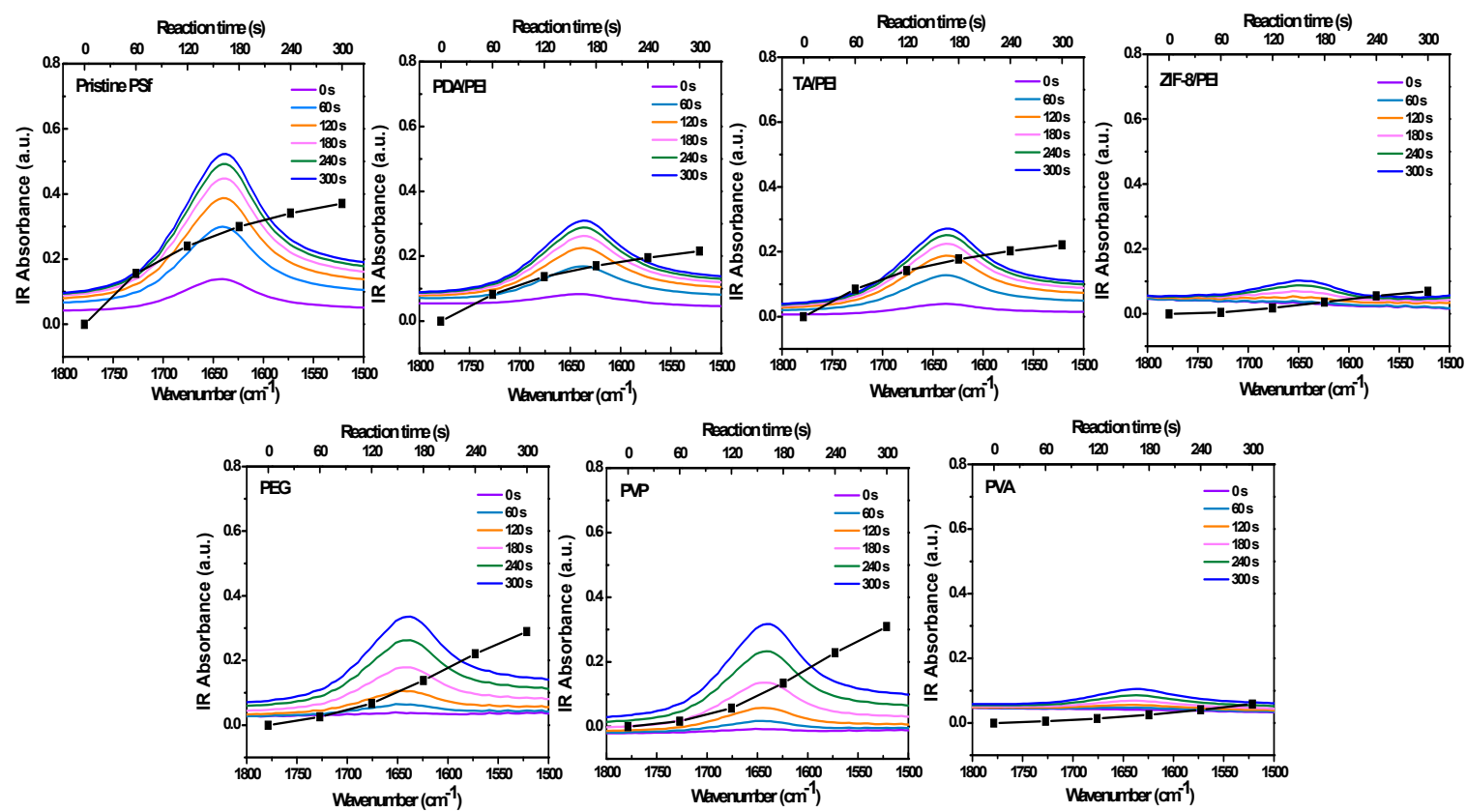

Figure 2. The in situ FT-IR spectra and absorbance intensities growth of polyamide formation process on porous substrate, which were characterized by the $\mathrm{C}=\mathrm{O}$ stretching vibration at $1640 \mathrm{~cm}^{-1}$.
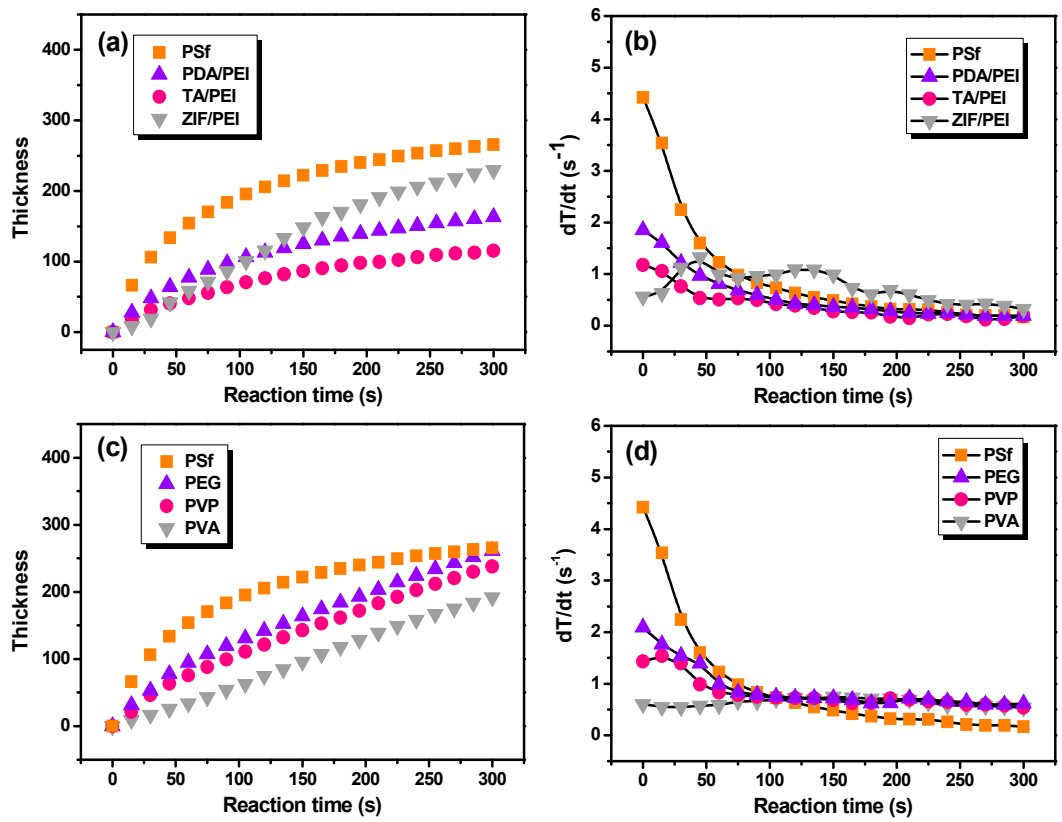

Figure 3. The growth of calculated polyamide layer thickness and the correspondingly polyamide layer thickness growth rate, as a function of IP reaction time including $(\mathbf{a}, \mathbf{b})$ IP reaction taking place on the porous substrates with various modified interlayers, $(\mathbf{c}, \mathbf{d})$ the doped polyamide layer with the macromolecular additives in the aqueous solution of PIP. 
It is still a huge challenge to measure polyamide layer thickness, which formed in situ on the porous substrate. Previously, various methods were used to deal with this issue, including reducing the IP reaction rate [11] or stopping the IP reaction at various times and then determining the formed film mass or thickness [12]. V. Freger's famous mathematical model predicts that the polyamide film thickness increases with the IP reaction time as $\mathrm{t}^{1 / 2}$, which is reported in the literature [36,37]. In this study, the in situ FT-IR absorbance intensities were related with the polyamide layer thickness. Figure 3 shows the growth rate of polyamide unitless thickness as a function of the IP reaction time. As a result, this method of FT-IR spectroscopy did not take variations in surface morphology of the polyamide into account, with this being another potential source of error on the thickness determination using FT-IR spectroscopy. Because there are questions regarding the vibration, the chemistry, the density, and the fact that in this study there was no validation on the FT-IR-based thickness, it would be better to use a unitless thickness in Figure 3. It can be seen that the IP reaction that took place on the pristine PSf substrate held the fastest film thickness increasing rate. In addition, the layer thickness of polyamide with the IP reaction time can be fitted as $X=24.8 \mathrm{t}^{1 / 2}$, where $X(\mathrm{~nm})$ is polyamide thickness and $t(\mathrm{~s})$ is the IP reaction time (Table S3 in the Supporting Information). Furthermore, the growth of polyamide layers thickness are mathematically described as $X=7.3 \mathrm{t}^{1 / 2}, 4.0 \mathrm{t}^{1 / 2}$, and $0.6 \mathrm{t}$ on the various substrates modified with PDA/PEI, TA/PEI, and ZIF-8/PEI interlayers, respectively (as shown in Figure 3a,b and Table S3 in the Supporting Information). At the same time, when the macromolecular additives were added in the aqueous solution of PIP (Figure 3c,d and Table S3 in the Supporting Information), the growth of polyamide thickness corresponded to $X=5.4 t^{2 / 3}, 3.3 t^{2 / 3}$, and $0.5 t$, respectively, which implied the repressed IP reaction rate by the introduction of macromolecular additives in the aqueous solution of PIP.

In the aim of confirming the accuracy and reliability of the in situ FT-IR spectroscopy method for the determination of the thickness of the polyamide layer, the TEM technique was employed, as shown in Figure 4 and Figure S12 in the Supporting Information. Figure 4 shows that the thickness of polyamide layers decreased noticeably with the introduction of interlayers and/or macromolecular additives. The ascertained thickness of polyamide layers obtained from the TEM images were at the sequence of $138 \pm 24 \mathrm{~nm}$ (PSf) $>95 \pm 7 \mathrm{~nm}(\mathrm{PEG})>84 \pm 17 \mathrm{~nm}(\mathrm{PVP})>76 \pm 19 \mathrm{~nm}(\mathrm{PVA})>$ $71 \pm 3 \mathrm{~nm}(\mathrm{PDA} / \mathrm{PEI})>60 \pm 6 \mathrm{~nm}(\mathrm{ZIF}-8 / \mathrm{PEI})>46 \pm 2 \mathrm{~nm}(\mathrm{TA} / \mathrm{PEI})$. Furthermore, the as-formed polyamide layer surface morphologies and the roughness were observed and evaluated by the FESEM images (Figure S13 in the Supporting Information) and the AFM images (Figure S14 and Table S4 in the Supporting Information). The obtained results showed that the polyamide surface morphologies maintained the "nodular" structures on the pristine PSf substrate and changed on the substrates modified with various interlayers, because the IP reaction happened directly on the interlayers and had different surface morphology features. Furthermore, with the ever-increasing ability of PVA $>$ PVP $>$ PEG to retard the PIP diffusion rate, the doped polyamide layers with macromolecular additives could generated the Turing structure, as reported in the literature [33].

Moreover, XPS analyses were used to investigate the elemental contents near the polyamide layer surface (shown in Table S5 in the Supporting Information). With regard to the traditional formed polyamide membrane on the PSf substrate, the polyamide layer retained the conventional "sandwich-structure", which meant the densest part was in the middle of the polyamide layer structure [36,37] and was loose on the top and bottom parts. In contrast, compared with the traditional polyamide structure, the polyamide layers formed on the pristine PSf substrates modified with various hydrophilic interlayers and led to the higher $\mathrm{O} / \mathrm{N}$ ratio and lower cross-linking degree (shown in Table S5 in the Supporting Information), further verifying the looser top structures of XPS penetration depth less than $10 \mathrm{~nm}$. Nevertheless, for doped polyamide layers with the macromolecular additives added in the aqueous PIP solution, the polyamide layer top structures were comparatively denser, with the lower $\mathrm{O} / \mathrm{N}$ ratio and higher cross-linking degree (shown in Table S5 in the Supporting Information) [38]. 

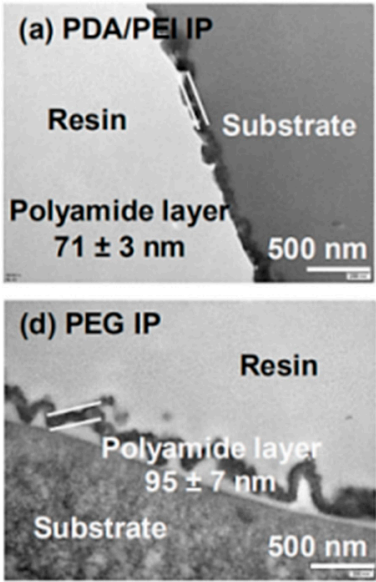
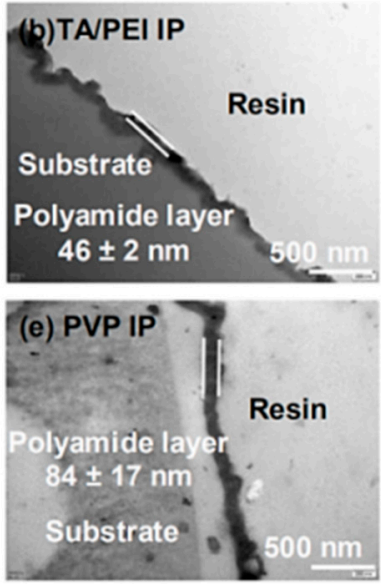
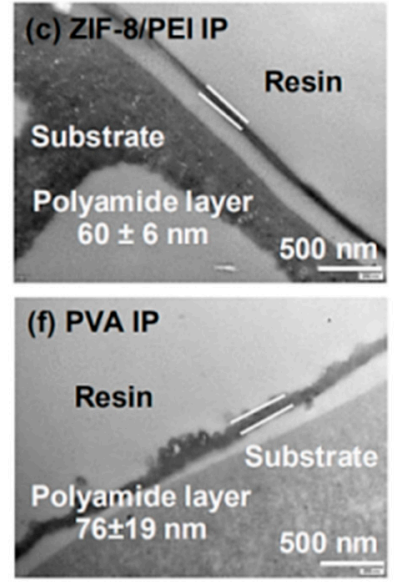

Figure 4. Transmission electron microscopy (TEM) observation of thickness of the formed polyamide layer on the $(\mathbf{a}-\mathbf{c})$ PSf substrates with various modified interlayers and $(\mathbf{d}-\mathbf{f})$ the doped polyamide layer with the macromolecular additives in the aqueous solution of PIP.

NF performances of the as-prepared polyamide membranes were evaluated by laboratory cross-flow equipment. The membranes' surface hydrophilicity were significantly improved by the introduction of interlayers and/or addition of macromolecular additives in the aqueous PIP solution, because of the potential changes of the polyamide surface groups. Moreover, polyamide membranes' surface charge were evaluated by the zeta potential analyzer (shown in Table S6 in the Supporting Information). The results of their surface charges indicated that NF membranes with the hydrophilic modified interlayers had higher surface charges compared with the doped polyamide membranes with macromolecular additives in the PIP solution.

It was noticeable that the NF performances, including the water permeation and inorganic salts rejection rate, were usually the synergistic results of the polyamide layer thickness and their internal cross-linking degree. With the aim of obtaining the high membrane water permeation, the polyamide layer thickness was expected to be as thin as possible. Otherwise, for achieving the high inorganic salts rejection rate, the membrane internal high cross-linking degree was necessarily required. In our experiments, after the introduction of hydrophilic interlayers or adding of the macromolecular additives in the aqueous PIP solution, water permeation enhancements were noteworthy, which was in the order of ZIF-8/PEI > TA/PEI > PDA/PEI, with the hydrophilic interlayers on the modified porous substrates, and PVA $>$ PVP $>$ PEG for the doped polyamide membranes with macromolecular additives (as shown in Figure 5a).
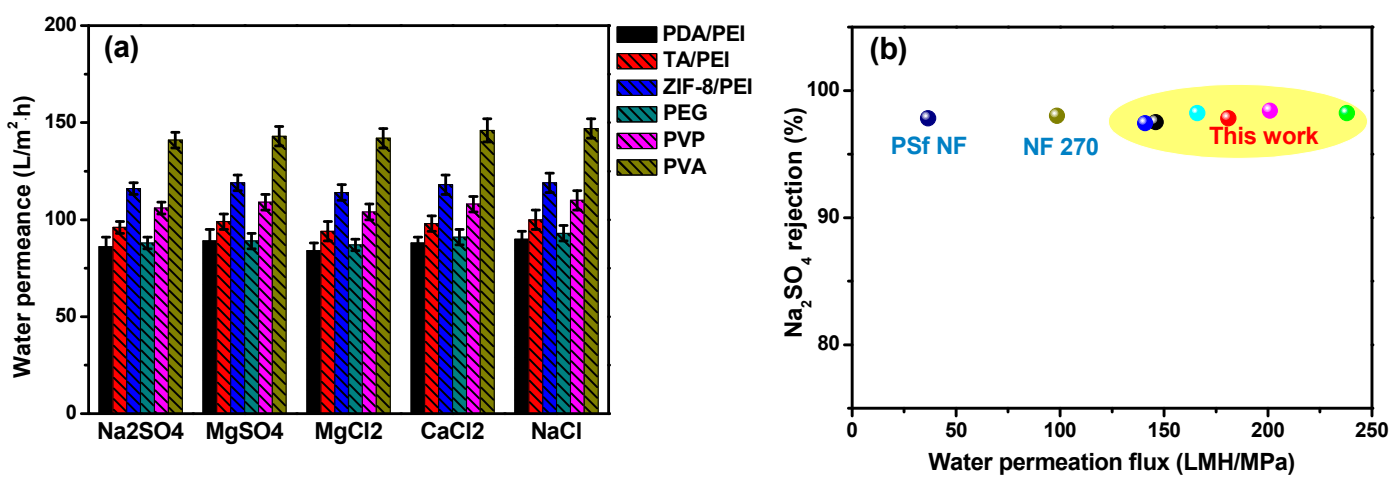

Figure 5. (a) Water permeation of the as-prepared NF membranes with the hydrophilic modified interlayers on the PSf substrates or the addition of macromolecular additives in the aqueous PIP solution (test conditions: inorganic salts concentration $=1000 \mathrm{mg} / \mathrm{L}, \mathrm{pH}=6.5,25^{\circ} \mathrm{C}$, applied pressure $=0.6 \mathrm{MPa}$ and cross-flow rate $=30 \mathrm{~L} / \mathrm{h}) ;(\mathbf{b})$ the comparison of the NF performance with other NF membranes in the reported literature $[30,39]$. 
Furthermore, inorganic salts' rejection of these NF membranes were presented at the order of $\mathrm{Na}_{2} \mathrm{SO}_{4}>\mathrm{MgSO}_{4}>\mathrm{MgCl}_{2}>\mathrm{CaCl}_{2}>\mathrm{NaCl}$ (Table S7 in the Supporting Information), which is in accordance with the typical NF membranes carried with the surface negative charges. In particular, the high rejections of $\mathrm{MgCl}_{2}$ and $\mathrm{CaCl}_{2}$ for these doped polyamide membranes with macromolecular additives could be attributed to the "compactly packing" of the macromolecular additives in the polyamide layer structures. By taking into account the combination of size sieving and Donnan exclusion effects, the hydrated radius of ions was at the order of $\mathrm{Mg}^{2+}(0.428 \mathrm{~nm})>\mathrm{Ca}^{2+}(0.412 \mathrm{~nm})>$ $\mathrm{Na}^{+}(0.358 \mathrm{~nm})$, and thus the resulting rejection of NF membranes was in the sequence of $\mathrm{MgCl}_{2}>$ $\mathrm{CaCl}_{2}>\mathrm{NaCl}[40,41]$. Furthermore, the electrostatic interaction between $\mathrm{SO}_{4}{ }^{2-}$ and the polyamide negative surface was stronger than $\mathrm{Cl}^{-}$, leading to the high rejections of $\mathrm{Na}_{2} \mathrm{SO}_{4}$ and $\mathrm{MgSO}_{4}$ (exceeding above $97.2 \%$ ). In summary, the as-prepared NF membranes provided a great opportunity to break out the "trade-off" behavior of the traditional polyamide membranes, showing high water permeation, and maintaining a superior inorganic salt rejection rate, thus having practical use in industry (as shown in Figure 5b).

\section{Conclusions}

To sum up, in situ FT-IR spectroscopy was for the first time employed to measure the IP reaction on the porous substrate. The results successfully validated the "depressed-effect" of both the introduced interlayers on the pristine PSf substrate and macromolecular additives in the PIP solution during the IP reaction. Though the exact forming mechanism of the dissimilar growing types of IP reaction were still not clear, the in situ FT-IR spectroscopy paved a new way in which to thoroughly comprehend the IP reaction mechanism. Furthermore, it could be utilized to tailor the superior performing thin-film composite membrane, consequently having significant and practical usage in the water treatment industry.

Supplementary Materials: The following are available online at http://www.mdpi.com/2077-0375/10/1/12/s1, Figure S1: ATR/FT-IR spectra of PSf substrates modified with interlayers; Figure S2. Surface morphologies observed by FESEM images of PSf substrate; Figure S3. XRD spectra of the synthesized ZIF-8 and ZIF-8/PEI; Figure S4. FESEM and EDX images of the ZIF-8/PEI modified PSf substrate; Figure S5. Water contact angle and zeta potential of PSf substrates; Figure S6. ATR/FT-IR spectra of the polyamide-based membranes formed on the substrates; Figure S7. Three-dimension spectra measured by in-situ FT-IR spectroscopy for the polyamide formation as a function of interfacial polymerization time; Figure S8. UV-vis absorption spectra of diamine monomer diffusion from water to hexane; Figure S9. Binding energies calculated between PIP and other molecules; Figure S10. Polyamide volume fractions converted from in-situ FT-IR spectra; Figure S11. Diamine diffusivity with the interfacial polymerization time, showing depressed and "self-limiting" effect; Figure S12. TEM observation of polyamide layer thickness observed on the pristine PSf substrate; Figure S13. FESEM images of surface morphologies of polyamide layers formed on the PSf substrates; Figure S14. AFM images of surface topographies of polyamide layers formed on the PSf substrates; Table S1. According to UV-vis adsorption spectra, the concentration and diffusivity of diamine monomers were determined, which were calculated by equation $\mathrm{S}$; Table S2. Adsorption mass of diamine monomers measured by TOC analyzer; Table S3. Polyamide layer thickness acquired from in-situ FT-IR spectroscopy; Table S4. Surface roughness of the membrane samples measured from AFM images; Table S5. XPS analyses of element component of the polyamide membrane surface and the corresponding calculated O/N ratio; Table S6. Water contact angle and zeta potential of the formed polyamide membrane; Table S7. Rejections of polyamide membrane for different kinds of inorganic salts.

Funding: This research received no external funding.

Acknowledgments: The author thanks Xiao-ying Gao for her help in manuscript revision, Zhe-Han Chen in the preparation of ZIF-8/PEI, Xin-Yu Lu in the experiments of in situ FT-IR spectroscopy, and Jing Yang's help for adding the macromolecular additives in the PIP solution in Department of Polymer Science \& Engineering, Zhejiang University.

Conflicts of Interest: The author declares no conflict of interest.

\section{References}

1. Elimelech, M.; Phillip, W.A. The future of seawater desalination: Energy, technology, and the environment. Science 2011, 333, 712-717. [CrossRef] 
2. Werber, J.R.; Osuji, C.O. Materials for next-generation desalination and water purification membranes. Nat. Rev. Mater. 2016, 1, 16018. [CrossRef]

3. Lv, Y.; Du, Y. Polymer nanofiltration membranes via controlled surface/interface engineering. Acta Polym. Sin. 2017, 12, 1905-1914.

4. Giagnorio, M.; Ricceri, F. Desalination of brackish groundwater and reuse of wastewater by forward osmosis coupled with nanofiltration for draw solution recovery. Water Res. 2019, 153, 134-143. [CrossRef] [PubMed]

5. Jin, L.; Wang, Z. Polyamide-crosslinked graphene oxide membrane for forward osmosis. J. Membr. Sci. 2018, 545, 11-18. [CrossRef]

6. Karan, S.; Jiang, Z. Sub-10 nm polyamide nanofilms with ultrafast solvent transport for molecular separation. Science 2015, 348, 1347-1351. [CrossRef]

7. Freger, V. Nanoscale heterogeneity of polyamide membranes formed by interfacial polymerization. Langmuir 2003, 19, 4791-4797. [CrossRef]

8. Zhang, X.; Lv, Y. Polyphenol coating as an interlayer for thin-film composite membranes with enhanced nanofiltration performance. ACS Appl. Mater. Interfaces 2016, 8, 32512-32519. [CrossRef]

9. Wang, J.-J.; Yang, H.-C. Nanofiltration membranes with cellulose nanocrystals as an interlayer for unprecedented performance. J. Mater. Chem. A 2017, 5, 16289-16295. [CrossRef]

10. Nowbahar, A.; Mansard, V. Measuring interfacial polymerization kinetics using microfluidic interferometry. J. Am. Chem. Soc. 2018, 140, 3173-3176. [CrossRef]

11. Ji, J.; Dickson, J.M. Mathematical model for the formation of thin-film composite membranes by interfacial polymerization: Porous and dense films. Macromolecules 2000, 33, 624-633. [CrossRef]

12. Bouchemal, K.; Couenne, F. Polyamides nanocapsules: Modeling and wall thickness estimation. AIChE J. 2006, 52, 2161-2170. [CrossRef]

13. Wagh, S.J.; Dhumal, S.S. An experimental study of polyurea membrane formation by interfacial polycondensation. J. Membr. Sci. 2009, 328, 246-256. [CrossRef]

14. Yadav, S.K.; Suresh, A.K. Microencapsulation in polyurea shell by interfacial polycondensation. AIChE J. 1990, 36, 431-438. [CrossRef]

15. Behera, S.; Suresh, A.K. Kinetics of interfacial polycondensation reactions-development of a new method and its validation. Polymer 2017, 127, 28-44. [CrossRef]

16. Chai, G.-Y.; Krantz, W.B. Formation and characterization of polyamide membranes via interfacial polymerization. J. Membr. Sci. 1994, 93, 175-192. [CrossRef]

17. Matthews, T.D.; Yan, H. Growth dynamics of interfacially polymerized polyamide layers by diffuse reflectance spectroscopy and Rutherford backscattering spectrometry. J. Membr. Sci. 2013, 429, 71-80. [CrossRef]

18. Mul, G.; Hamminga, G.M. Operando ATR-FTIR analysis of liquid-phase catalytic reactions: Can heterogeneous catalysts be observed? Vib. Spectrosc. 2004, 34, 109-121. [CrossRef]

19. Hind, A.R.; Bhargava, S.K. At the solid/liquid interface: FTIR/ATR-the tool of choice. Adv. Colloid Interface Sci. 2001, 93, 91-114. [CrossRef]

20. Sawunyama, P.; Jiang, L. Photodecomposition of a Langmuir-Blodgett film of stearic acid on $\mathrm{TiO}_{2}$ film observed by in situ atomic force microscopy and FT-IR. J. Phys. Chem. B 1997, 101, 11000-11003. [CrossRef]

21. Andrew Chan, K.L.; Kazarian, S.G. FT-IR spectroscopic imaging of reactions in multiphase flow in microfluidic channels. Anal. Chem. 2012, 84, 4052-4056. [CrossRef] [PubMed]

22. Han, J.; He, Y. Photopolymerization of alicyclic methacrylate hydrogels for controlled release. Polym. Adv. Technol. 2009, 20, 607-612. [CrossRef]

23. Zimudzi, T.J.; Hickner, M.A. Signal enhanced FTIR analysis of alignment in Nafion thin films at $\mathrm{SiO}_{2}$ and $\mathrm{Au}$ interfaces. ACS Macro Lett. 2016, 5, 83-87. [CrossRef]

24. Zimudzi, T.J.; Feldman, K.E. Quantifying carboxylic acid concentration in model polyamide desalination membranes via Fourier transform infrared spectroscopy. Macromolecules 2018, 51, 6623-6629. [CrossRef] [PubMed]

25. Singh, P.S.; Rao, A.P. Techniques for characterization of polyamide thin film composite membranes. Desalination 2011, 282, 78-86. [CrossRef]

26. Ohta, K.; Iwamoto, R. Experimental proof of the relation between thickness of the probed surface layer and absorbance in FT-IR/ATR spectroscopy. Appl. Spectrosc. 1985, 39, 418-425. [CrossRef]

27. Jin, Y.; Su, Z. Effects of polymerization conditions on hydrophilic groups in aromatic polyamide thin films. J. Membr. Sci. 2009, 330, 175-179. [CrossRef] 
28. Singh, P.S.; Joshi, S.V. Probing the structural variations of thin film composite RO membranes obtained by coating polyamide over polysulfone membranes of different pore dimensions. J. Membr. Sci. 2006, 278, 19-25. [CrossRef]

29. Ren, D.; Yeo, J.I.N. Time-dependent FTIR microscopy for mechanism investigations and kinetic measurements in interfacial polymerisation: A microporous polymer film study. Polym. Chem. 2019, 10, 2769-2773. [CrossRef]

30. Yang, X.; Du, Y. Nanofiltration membrane with a mussel-inspired interlayer for improved permeation performance. Langmuir 2017, 33, 2318-2324. [CrossRef]

31. Yang, X. Controllable interfacial polymerization for nanofiltration membrane performance improvement by the polyphenol interlayer. ACS Omega 2019, 4, 13824-13833. [CrossRef] [PubMed]

32. $\mathrm{Wu}, \mathrm{X}$; $\mathrm{Li}$, Y. Adsorption-assisted interfacial polymerization toward ultrathin active layers for ultrafast organic permeation. ACS Appl. Mater. Interfaces 2018, 10, 10445-10453. [CrossRef] [PubMed]

33. Tan, Z.; Chen, S. Polyamide membranes with nanoscale Turing structures for water purification. Science 2018, 360, 518-521. [CrossRef]

34. Yang, L.; Wang, Z. Highly permeable zeolite imidazolate framework composite membranes fabricated via a chelation-assisted interfacial reaction. J. Mater. Chem. A 2017, 5, 15342-15355. [CrossRef]

35. Yang, L.; Wang, Z. Zeolite imidazolate framework hybrid nanofiltration (NF) membranes with enhanced permselectivity for dye removal. J. Membr. Sci. 2017, 532, 76-86. [CrossRef]

36. Freger, V.; Srebnik, S. Mathematical model of charge and density distributions in interfacial polymerization of thin films. J. Appl. Polym. Sci. 2003, 88, 1162-1169. [CrossRef]

37. Freger, V. Kinetics of film formation by interfacial polycondensation. Langmuir 2005, 21, 1884-1894. [CrossRef]

38. Kong, X.; Qiu, Z.-L. High permselectivity hyperbranched polyester/polyamide ultrathin films with nanoscale heterogeneity. J. Mater. Chem. A 2017, 5, 7876-7884. [CrossRef]

39. Hu, R.; Zhang, R. Graphene oxide-in-polymer nanofiltration membranes with enhanced permeability by interfacial polymerization. J. Membr. Sci. 2018, 564, 813-819. [CrossRef]

40. Lin, C.-E.; Fang, L.-F. A novel positively charged nanofiltration membrane formed via simultaneous cross-linking/quaternization of poly (m-phenylene isophthalamide)/polyethyleneimine blend membrane. Sep. Purif. Technol. 2019, 212, 101-109. [CrossRef]

41. Fang, L.-F.; Zhou, M.-Y. Positively charged nanofiltration membrane based on cross-linked polyvinyl chloride copolymer. J. Membr. Sci. 2019, 572, 28-37. [CrossRef] 